\title{
Cardiovascular disease and the UN Millennium Development Goals: time to move forward
}

\author{
Valentin Fuster and Rajesh Vedanthan
}

It is now well-established that cardiovascular disease (CVD) is the leading cause of death worldwide and that the burden of CVD is greatest in low-income and middle-income countries. According to the WHO, in 2005 there were 58 million deaths globally, of which 35 million were caused by chronic diseases. Over $80 \%$ of these deaths resulting from chronic disease occurred in low-income and middle-income countries, and CVD-related mortality comprised the largest proportion of deaths in these regions.

Despite these statistics, CVD and other chronic diseases have been under-recognized and their treatment under-funded when compared with infectious diseases such as HIV/ AIDS, tuberculosis and malaria. One of the factors contributing to this lack of recognition was the UN's Millennium Development Goals (MDGs) project, an issue which was highlighted in this journal back in August 2006 (Fuster V [2006] Nat Clin Pract Cardiovasc Med 3: 401). From the initial conception of these goals there was inadequate representation of chronic diseases. The primary health foci of the MDGs were the reduction of child and maternal mortality, and the prevention of the spread of HIV/AIDS, malaria and other diseases. Notably absent from this formulation were the chronic diseases, including CVD.

Fortunately, the situation is changing for the better. The recognition of the epidemiologic and economic burden of chronic diseases including CVD has increased in recent years. This change in awareness has led to new initiatives at various levels: new national and international policies addressing obesity, the use of tobacco products and other cardiovascular risk factors; increased research funding and a proliferation of opportunities for faculty development promoted by the NIH and private foundations; and the development of clinical and public health programs targeting CVD by international aid agencies and local governments.
The recognition

of the

epidemiologic

and economic

burden of

chronic

diseases

including CVD

has increased

in recent years.

$V$ Fuster is the Editorin-Chief of Nature

Clinical Practice

Cardiovascular

Medicine.

$R$ Vedanthan

is a Fellow in

Cardiovascular

Medicine at the

Mount Sinai Medical

Center in New York,

NY, USA.

\section{Competing interests}

The authors declared no

competing interests.

www.nature.com/clinicalpractice doi:10.1038/ncpcardio1353
Developed in collaboration with Jeffrey Sachs, one of the pioneers of the MDGs, one of these new developments includes the Millennium Villages Project (MVP) CVD Initiative. The MVP is based in 11 sub-Saharan African countries and is a proof-of-concept project to demonstrate that an integrated package of interventions - increased food production, access to clean water, sanitation, education, infrastructure development and easy access to health care — can assist people living in extreme poverty and lay the path for economic development. In 2006, a survey in three of the participating villages revealed a higher-than-anticipated incidence of hypertension and smoking, and it was decided that chronic and noncommunicable diseases could no longer be neglected. Thus, the MVP CVD Initiative is developing a program to quantify more comprehensively the local prevalence of cardiovascular risk factors, understand the subtleties of local conceptions of CVD, and subsequently implement both individual-level and population-level interventions to decrease CVD risk factors and ultimately the burden of CVD.

Initiatives such as these are positive steps towards including chronic diseases in the MDGs. In addition, these programs strengthen and broaden health-care systems in resourcepoor settings. Interpretation of the MDGs has frequently meant that health-care issues other than infectious diseases are largely ignored in low-income countries. Fortunately, this situation is being rectified at multiple levels. As initiatives such as the MVP continue to evolve, they will benefit from a constructive engagement between the infectious disease and chronic disease communities. The lessons learned about delivering efficient health care on a global scale, gained in the context of HIV, tuberculosis and malaria programs, should be applied to fighting CVD. As we move forward, these lessons will help us tackle what otherwise threatens to be an overwhelming burden of disease. 